\title{
Noninvasive visualization of early osteoarthritic cartilage using targeted nanosomes in a destabilization of the medial meniscus mouse model
}

This article was published in the following Dove Press journal:

International Journal of Nanomedicine

\author{
Hongsik Cho ${ }^{1,2, *}$ \\ Byoung Ju Kim ${ }^{3,4, *}$ \\ Sang-Hyug Park ${ }^{5}$ \\ Karen A Hasty ${ }^{1,2}$ \\ Byoung-Hyun Min 3 3,46 \\ 'Department of Orthopaedic Surgery \\ and Biomedical Engineering, University \\ of Tennessee Health Science Center- \\ Campbell Clinic, ${ }^{2}$ Veterans Affairs \\ Medical Center, Memphis, TN, USA; \\ ${ }^{3}$ Department of Molecular Science \\ and Technology, Ajou University, ${ }^{4}$ Cell \\ Therapy Center, Ajou University \\ Hospital, Suwon, ${ }^{5}$ Department of \\ Biomedical Engineering, Pukyong \\ National University, Nam-Gu, Busan, \\ ${ }^{6}$ Department of Orthopedic Surgery, \\ School of Medicine, Ajou University, \\ Suwon, Republic of Korea
}

*These authors contributed equally to this work

Correspondence: Byoung-Hyun Min Department of Orthopedic Surgery, Ajou University School of Medicine, I 64 Worldcup-ro, Yongtong-gu, Suwon 16499, Republic of Korea

Tel +82312195225

Fax +82 31 2191504

Email bhmin@ajou.ac.kr

Karen A Hasty

Department of Orthopaedic Surgery and Biomedical Engineering University of Tennessee Health Science CenterCampbell Clinic Research Service I5I, VA Medical Center, 1030 Jefferson Avenue, Memphis, TN 38104, USA

$\mathrm{Tel}+\mathrm{I} 9015238990$ ext 6175

Fax + I 90I 5777273

Email khasty@uthsc.edu
Background: Early stage osteoarthritis (OA) is clinically asymptomatic due to the avascular and the aneural nature of the cartilage tissue. Nevertheless, early detection of cartilage tissue is critical in order to impede the progression of OA. Hence, in order to develop effective preventive therapy for OA, diagnosis in the early stages is necessary.

Methods: To achieve this goal, we have developed targeted, fluorescent nanosomes conjugated with monoclonal anti-type II collagen antibodies (MabCII) for diagnosis of early OA. The MabCII-coated nanosomes (targeted-nanosomes) bind to the damaged cartilage explants in vitro and in vivo in an OA mouse model that mimics early stage OA. The OA mouse model was induced by destabilization of the medial meniscus (DMM) in 9-10 weeks old C57B1/6 mice.

Results: The targeted-nanosomes enhanced the binding specificity to the cartilage tissue according to the severity of damage.

Conclusion: We show that MabCII-nanosomes can precisely detect early stage OA in the DMM mouse model. Thus, MabCII-nanosomes have the potential to be used as a non-invasive method for diagnosing the early osteoarthritic lesions.

Keywords: osteoarthritis, nanosome, diagnosis, OA score, destabilization of the medial meniscus, matrix metalloproteinases, monoclonal anti-type II collagen antibody

\section{Introduction}

Osteoarthritis (OA) is an extremely common type of arthritis, which is one of the leading causes of disability in the world. ${ }^{1} \mathrm{OA}$ is a manifestation of obesity, aging, trauma, and mechanical stress. ${ }^{2,3}$ It typically appears in weight-bearing joints as focal lesions that progressively deepen until the subchondral bone is exposed..$^{4,5}$ Although focal lesions in the cartilage can be repaired, no appropriate treatment has been developed to reverse cartilage degradation. Thus, the best strategy is to diagnose OA in its early stages and hence prevent the total loss of cartilage tissue. ${ }^{6}$ Diagnosis of early-stage OA is difficult because of its asymptomatic nature as the patients do not recognize pain due to the aneural nature of cartilage. ${ }^{7,8}$ As a result, early lesions are not painful and often go undetected until the damage is irreversible. In general, diagnosis relies on the radiographic or arthroscopic evaluation of the articular surface, which only detects macroscopic damage to the cartilage. ${ }^{7-9}$ Histological observation is the most accurate diagnostic method, but its invasiveness limits its application to animal experiments. Although magnetic resonance imaging (MRI) has been proposed as a 
method of choice for noninvasive diagnosis, MRI still fails to detect International Cartilage Repair Society grade level $1 \mathrm{OA} \cdot{ }^{10-12}$ It might be possible to diagnose OA by measuring the presence of OA-specific biomarkers in body fluids, but the biomarkers are not specific to joint tissues alone. ${ }^{14,15}$ In addition, their concentrations are often altered by physical activity or food consumption, resulting in readings that can lead to poor diagnosis of the disease state. ${ }^{10}$

The earliest lesions in joint cartilage result from the destruction of the cartilage extracellular matrix (ECM), which is mainly composed of collagen and proteoglycan. ${ }^{11,12}$ The lesions weaken the cartilage and reduce its capacity to withstand external load, ultimately causing increased cartilage wear. Therefore, it is crucial to look for changes in the ECM to detect cartilage lesions early on. ${ }^{12}$ Various studies are underway to identify methods to detect lesions such as direct observation of damaged ECM, injection of a substituting substance for lost components of the matrix, and observation of substances secreted outside the joint cartilage by degradation of the ECM damage..$^{13,14}$

An optical molecular imaging technique has gained considerable attention due to its unique ability to monitor the dynamic extracellular composition in real time. It has been used to test body fluids for the presence of biomarkers such as glycosaminoglycan (GAG) and collagen ${ }^{15-17}$ or their degradative complex including zinc(II) dipicolyamine, ${ }^{18}$ cathepsin $\mathrm{B},{ }^{19}$ and matrix metalloproteinase $13 .{ }^{20}$

Nanoscaled liposomes called nanosomes serve as an effective targeted drug delivery system. Here, we present a method for early diagnosis of $\mathrm{OA}$ in vivo and serial measurement of cartilage damage in individual joints using type II collagen (CII)-targeted nanosomes. ${ }^{21}$ Our technique uses nanosomes that are targeted to exposed CII using a monoclonal antibody (Mab). ${ }^{21}$ It was initially shown by Jasin and coworkers that normal articular cartilage poses a barrier to the binding of antibodies. ${ }^{3,4,22}$ However, when the surface of the cartilage is exposed by proteolytic enzymes, the native CII is exposed. The exposed CII is then accessible to anti-CII antibodies. ${ }^{22-24}$ Our targeted nanosomes are incorporated with an anti-CII Mab and a near-infrared fluorescent (NIF) dye that can be visually quantified by using an external imaging system called IVIS (IVIS ${ }^{\circledR}$ Lumina XR System; PerkinElmer Inc., Waltham, MA, USA). ${ }^{21,25}$ Furthermore, the noninvasive method developed in this study can be used to detect cartilage damage existing in early-stage OA as proven by comparable results obtained through histopathological examination.

\section{Materials and methods \\ Experimental animal and surgical induction of $O A$}

\section{Animals}

Thirty-six male C57BL/6 mice ( $\mathrm{n}=36$; 9-10 weeks old, 22-24 g) were purchased from Orient Bio Inc. (Seongnam, South Korea). All animals were housed in the same facility on a 12-hour light/dark cycle. The mice had ad libitum access to feed and tap water. Thirty of the animals underwent destabilization of the medial meniscus (DMM) surgery, and six animals underwent sham surgery (Table 1), as described later. Animals were cared for according to institutional animal care and use protocols, and all animal studies were approved by the Animal Care and Use Committee of Ajou (Suwon, South Korea), University School of Medicine (IRB No AMC118).

\section{Surgical induction of $\mathrm{OA}$}

The surgical OA mouse model was induced by scalpel incision of the cranial meniscotibial ligament of the medial meniscus in the right knee joint of the hind limb as described previously. ${ }^{26-28}$ All animals were anesthetized with a mixture of Zoletil (50 mg/kg), Rompun (10 mg/kg), and saline. The sham-operated controls underwent the same surgery except that the meniscotibial ligament was not cut. Mice were allowed free cage activity after surgery and ad libitum for 3 , 7, 14, 21, and 28 days after surgery (Figure 1). Later, mice were euthanized at day 1 following retro-orbital injection of targeted nanosomes.

\section{Setup of the experimental group}

Data from the experimental groups were arranged according to OA severity (average of OA score) by using a modified

Table I Classification of the experimental groups of the study

\begin{tabular}{|c|c|c|c|c|c|c|}
\hline & \multirow{2}{*}{$\begin{array}{c}\text { Sham } \\
\text { A }\end{array}$} & \multicolumn{5}{|c|}{ Experimental groups } \\
\hline & & B & C & D & $\mathbf{E}$ & $\mathbf{F}$ \\
\hline OA score & $0.20 \pm 0.041$ & $0.79 \pm 0.094$ & $1.68 \pm 0.217$ & $2.26 \pm 0.109$ & $2.64 \pm 0.189$ & $3.33 \pm 0.393$ \\
\hline
\end{tabular}

Notes: Classification of the experimental groups according to the average OARSI score in specific range per group ( $\mathrm{n}=6$ per group). (A) Sham and (B-F) mice were grouped according to the average OARSI score and not according to days postsurgery. This classification was followed for further observation in this study.

Abbreviations: OA, osteoarthritis; OARSI, Osteoarthritis Research Society International. 


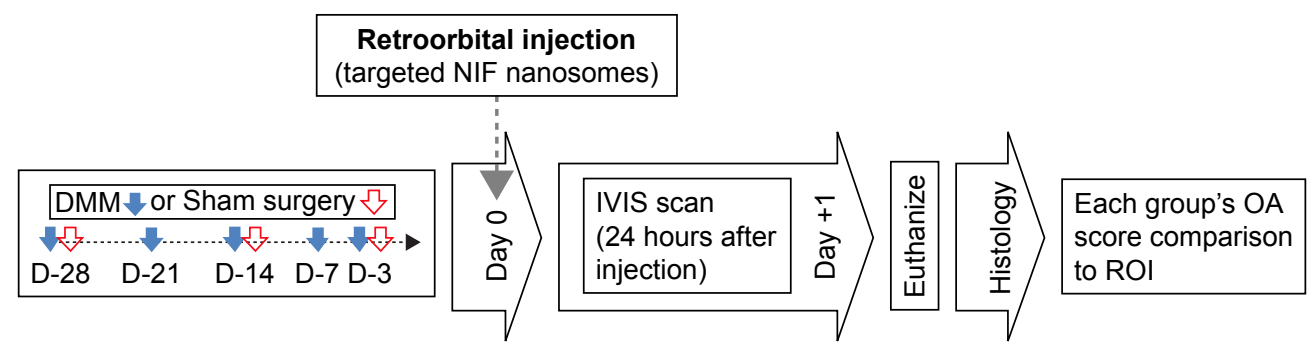

Figure I Experimental timeline.

Notes: DMM surgeries were performed 3, 7, 14, 2I, and 28 days before injection of targeted nanosomes. The time point of injecting targeted nanosomes was set as day 0. Mice were scanned 24 hours after injection (day $+\mathrm{I}$ ) and then euthanized (sham surgeries were performed 3 , I4, and 28 days before injection of targeted nanosomes). Abbreviations: DMM, destabilization of the medial meniscus; IVIS, in vivo imaging system; NIF, near-infrared fluorescent; OA, osteoarthritis; ROI, region of interest.

Osteoarthritis Research Society International (OARSI) scoring system (Table 1). ${ }^{21,26,27}$ Briefly, mice were divided into six distinctive groups with group A serving as sham surgery control. The OA groups were classified on the basis of increasing OA severity in each respective group (B-F groups in Table 1). No histopathological change was observed in the sham surgery group during the same time interval. The average OARSI scores were compared with the region of interest (ROI; calculated radiant efficiency) values from IVIS scanning to measure the correlation between OARSI score and the fluorescent intensity.

\section{Preparation of nanosomes}

\section{Target materials}

To target the damaged articular surface, we used a monoclonal anti-CII antibody from the VA Program Project Scientific Core at the VA Medical Center (Memphis, TN, USA). The generation and characterization of the anti-CII monoclonal antibodies are described in Terato et al. ${ }^{29}$ Briefly, an immunoassay was performed to measure binding and specificity of these antibodies for CII purified from mouse cartilage. The results showed that monoclonal anti-CII antibody (MabCII) (D1), the antibody chosen for this study, had the strongest immune reactivity and that this antibody recognized the amino acid sequence CII (124-290). ${ }^{29}$ The monoclonal mouse immunoglobulin G (IgG)2A antibody (MAbCon) of the same subclass (R\&D Systems, Inc., Minneapolis, MN, USA) served as the control.

\section{Nanosome chemistry}

Nanosomes were prepared from lipid films at a ratio of $5.2 \mu \mathrm{mol}$ 1,2-dioleoyl-sn-glycero-3-phosphocholine (DOPC), $4.5 \mu \mathrm{mol}$ cholesterol, $0.3 \mu \mathrm{mol}$ (DSPE-PEG2000), and $0.015 \mu \mathrm{mol}$ 1,2-distearoyl-sn-glycero-3-phosphoethanolamine- $N$-[maleimide (polyethylene glycol) 2000] (DSPE-PEG2000-maleimide), initially dried under a nitrogen stream, and then further dried under vacuum. All lipids were from Avanti Polar Lipids (Alabaster, AL, USA). The lipid film was rehydrated with Xenofluor 680 and multilamellar vesicles extruded through polycarbonate track-etched filters with uniform cylindrical pores averaging $200 \mathrm{~nm}$, resulting in unilamellar nanosomes with an average diameter of $200 \mathrm{~nm}$. Free dye was separated by gel filtration chromatography. Antibodies were thiolated and attached to the DSPE-PEG2000-maleimide moiety of the nanosomes and isolated from the free antibody by gel filtration. ${ }^{21,30-32}$ For the control nanosomes, the antibody was not thiolated, but the antibody was mixed with the nanosomes. Equivalent size distribution, NIF labeling, phospholipid content, and antibody labeling among the different nanosome preparations were confirmed by transmission electron microscopy (TEM), fluorometry, and dynamic light scattering (DLS). Binding efficacy of the MabCII nanosomes to CII was confirmed by CII ELISA. Briefly, 96-well plates were coated with $1 \mu \mathrm{g}$ of denatured CII per well and then blocked with $1 \%$ bovine serum albumin. All samples, whether the NIF nanosomes with MabCII or free antibody, were incubated at $4{ }^{\circ} \mathrm{C}$ overnight, followed by a phosphate-buffered saline wash. The fluorescence in nanosomes that bound CII was measured by fluorometer (SpectraMax M5; Molecular Devices LLC, Sunnyvale, CA, USA).

\section{Treatment of cartilage explants with fluorescent nanosomes}

Targeted nanosomes were tested on cartilage explants obtained from the knee joints of 2- to 3-month-old healthy pigs recently euthanized for other experiments according to the approved protocols and experimental procedures at the University of Tennessee Health Science Center. To mimic various degrees of damaged cartilage, the harvested cartilage pieces were treated with $200 \mu \mathrm{L}$ of different concentrations $(0,0.1,0.5$, and $1.25 \%)$ of Trypsin-EDTA (Thermo 
Fisher Scientific, Waltham, MA, USA) for 30 minutes at $37^{\circ} \mathrm{C}$. The explants were washed with $1 \%$ fetal calf serum, and $4.6 \times 10^{11} \mathrm{ea} / \mathrm{mL}$ of targeted nanosome particles were washed with encapsulated NIF fluorescent dye (XenoLight CF680; PerkinElmer Inc.) were added. After incubation for 1 hour at room temperature, the explants were washed with $1 \times$ phosphate-buffered saline. From each cartilage piece, 4-mm explants were punched out using a biopsy punch (Integra Miltex, Plainsboro, NJ, USA). The explants were scanned with the IVIS imaging system according to the manufacturer's instructions for XenoLight CF680.

\section{Fluorescence imaging analysis}

MabCII-targeted nanosomes encapsulating the NIF fluorescent dye were injected retro-orbitally ( $1 \mu \mathrm{g}$ of MabCII/g body weight; $100 \mu \mathrm{L}$ of targeted nanosomes) in the sham or DMM mice group at 1, 2, and 4 weeks postoperatively. At each week of the time interval, animals were anesthetized with $2.5 \%$ isoflurane 24 hours after injection of the final probe and scanned by IVIS imaging to determine fluorescent binding in the knee joint. The fluorescence from the knee joint was quantified by the Living Image software to calculate the flux radiating omnidirectionally from the ROI and graphed as radiant efficiency ([photons $\left./ \mathrm{second} / \mathrm{cm}^{2} / \mathrm{str}\right] /\left[\mu \mathrm{W} / \mathrm{cm}^{2}\right]$ ). To determine an average ROI from the knee fluorescence, the same area of capture was used for each mouse. Fluorescence from a background capture area (consisting of muscle and skin tissue) was measured and subtracted from each fluorescence reading of knee area. ${ }^{21}$ The knee joints of the euthanized mice were rescanned after dissection to confirm the absence of nonspecific binding on tibial, femoral, or patellar cartilage. We also used noninvasive fluorescence imaging system (Fluobeam; Fluoptics, Grenoble, France) to investigate early (1-3 hours) biodistribution and trace of the MabCII nanosomes in mice after injection. To scan early distribution of the nanosomes, the mouse was skinned to make the signal clearer.

\section{Histopathology}

Experimental groups of mice were euthanized with carbon dioxide at 1, 2, and 4 weeks after surgery. The right knee joints of the mice were separated and fixed in $4 \%$ paraformaldehyde for 24 hours. Whole joint tissue was decalcified in 5\% nitric acid for 10 hours at room temperature. Tissue cassettes were placed in formalin, dehydrated in a graded series of ethanol and xylene, and embedded in paraffin wax. Tissue blocks were cut into serial coronal sections of $5 \mu \mathrm{m}$ thickness at $100 \mu \mathrm{m}$ intervals. The sections were stained with hematoxylin/eosin to observe tissue histology and Safranin-O/
Fast Green stain to examine the amount of sulfated GAGs, as described previously. ${ }^{21,28}$ Coronal sections, from patella to posterior condyles, resulted in 25 sections. We selected three slides from the middle of the sections. The Safranin-O/Fast Green staining images of medial tibial cartilage were used to determine OA status of samples by the OARSI scale with slight modifications as described in the previous study. ${ }^{21,27,28}$ Scoring was done by two independent researchers, and results were averaged. Each investigator was blinded to the scores of the other investigator. The maximum score of the three sections was taken as the representative score of the knee joint. Histological scoring was performed on the medial tibial plateaus of Safranin-O/Fast Green staining images.

\section{Statistics}

Data were presented in histograms as mean \pm SD from six individual samples $(n=6)$. Statistical analysis was performed by the analysis of Student's $t$-tests (each group compared with their control group or nontreatment group). All experiments were performed independently at least three times. The degree of correlation between cartilage degeneration and binding of fluorescent nanosome within the medial knee compartments of the OA-induced mice was measured using Pearson's correlation coefficient $(r)$ test method $(* P<0.05$ and $* * P<0.01)$.

\section{Results \\ Characterization of nanosomes}

In our preliminary studies, we have tested and characterized our diagnosis and delivery system using the MabCII antibody-coated nanosomes (MabCII nanosomes) to target damaged cartilage explants in vitro or in vivo in DMM mice. The liposomes are easily identified as predominantly spherical discrete particles, with one to three membrane lamellae. Optical measurement showed that the size of particles ranged from 100 to $300 \mathrm{~nm}$ in diameter (Figure 2A). This measurement is consistent with the size distribution seen in the DLS data, which yielded one narrow peak of particles centered around the $100 \mathrm{~nm}$ radius (Figure 2B). Furthermore, the CII ELISA showed that only MabCII nanosomes have significantly high relative fluorescence when compared with either nanosomes without antibody or nanosomes with control antibody (Figure 2C). This shows that MabCII nanosomes have a high affinity for CII, and hence, they can bind specifically to the exposed CII both in vitro and in vivo.

\section{In vitro binding efficacy of nanosomes}

We evaluated the binding efficacy of our MabCII-targeted nanosomes to tissue, according to the degree of cartilage 


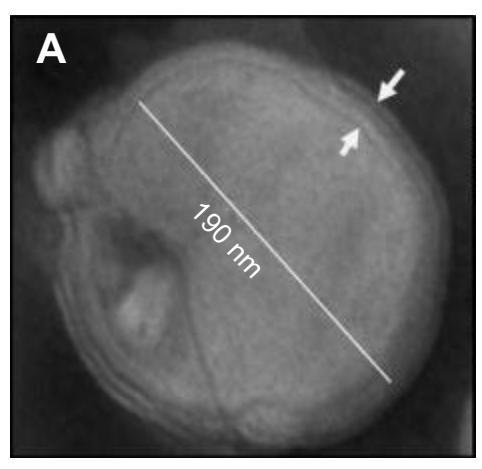

B

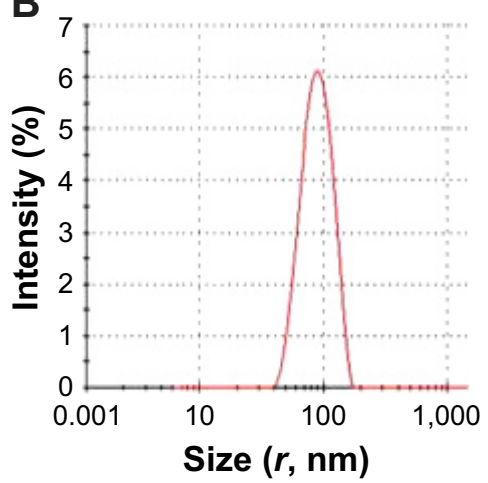

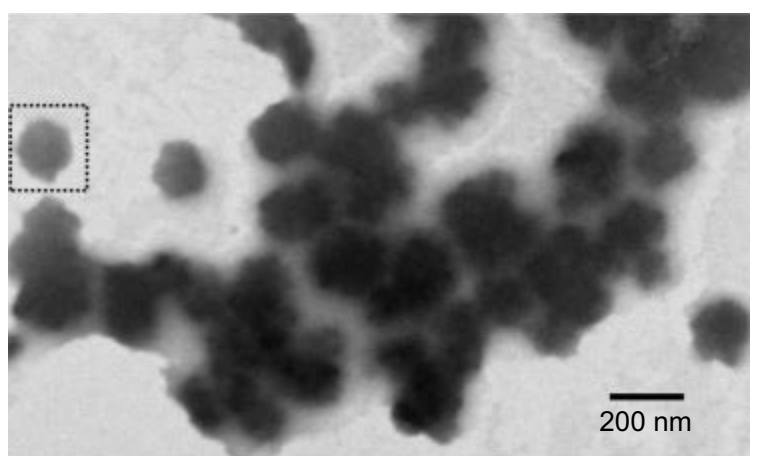

C

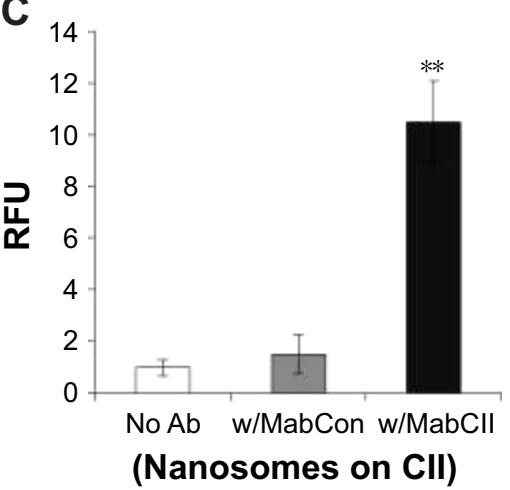

Figure 2 Characterization of targeted nanosomes.

Notes: (A) TEM images of nanosomes. Nanosome size and shape were confirmed by TEM. The nanosomes were multilamellar structures with I-3 layers. After coupling to antibody, the shape and size of the liposomes remain constant. Arrows indicate the lipid bilayer. (B) Size distribution measured using DLS. Nanosomes are uniform in size with average radius of $100 \mathrm{~nm}$. (C) CII ELISA of three different treatment groups (No Ab: nanosomes without antibodies, w/MabCon: nanosomes labeled with monoclonal mouse IgG, and w/MabCll: nanosomes labeled with anti-type II collagen antibodies). MabCll nanosomes showed significant binding to Cll. **P<0.0I.

Abbreviations: Cll, type II collagen; DLS, dynamic light scattering; IgG, immunoglobulin G; MabClI, monoclonal anti-type II collagen antibodies; RFU, relative fluorescence units; TEM, transmission electron microscopy.

damage. Different concentrations $(0,0.1,0.5$, and $1.25 \%)$ of trypsin were administered to porcine cartilage explants to simulate varying degrees of cartilage damage. Trypsinized explants were treated with MabCII-targeted nanosomes, control nanosomes with nonspecific antibody (MabCon: control antibodies; same subclass mouse $\operatorname{IgG}$ ) to CII, or nanosomes alone. Only MabCII-targeted nanosomes were observed to bind the damaged cartilage. Fluorescence intensity due to binding of the targeted nanosomes increased with trypsin-induced damage (Figure 3A). The NIF MabCon nanosomes and the nanosomes without antibody did not bind to the trypsinized cartilage, reinforcing the specificity of MabCII nanosomes. ROI readings were used to quantify the degree of fluorescent nanosome binding (Figure 3B).
A

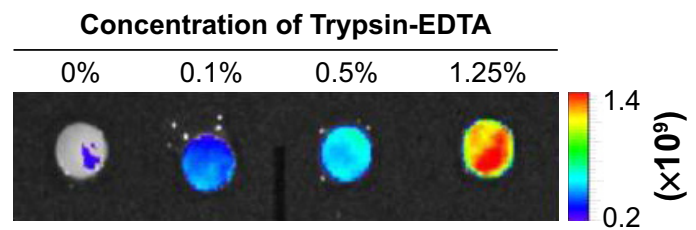

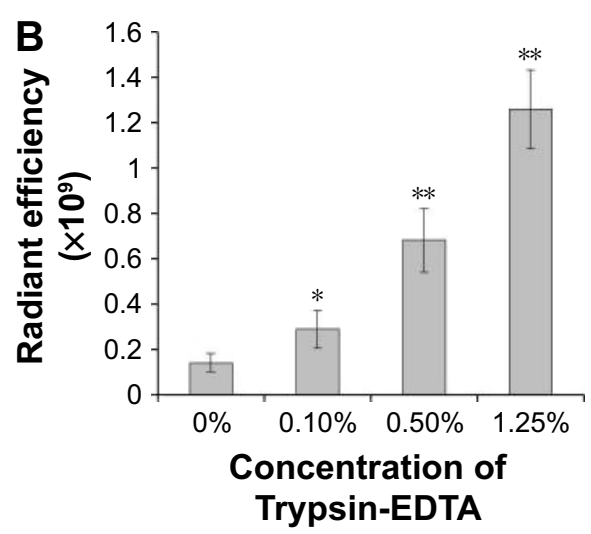

Figure 3 Binding efficacy of MabCll-targeted nanosomes to damage cartilage.

Notes: (A) Pig cartilage explants show the binding specificity of the nanosomes according to the severity of damage using IVIS. The explants were treated with different concentrations $(0,0.1,0.5$, and $1.25 \%)$ of Trypsin-EDTA. (B) ROI quantification was performed to measure the binding of fluorescent nanosomes ( $* P<0.05$ and $* * P<0.01)$.

Abbreviations: IVIS, in vivo imaging system; MabCll, monoclonal anti-type II collagen antibodies; ROI, region of interest. 


\section{In vivo targeting of MabCll-targeted- nanosomes}

The sham-operated knee did not display any binding of the NIF-MabCII or binding of the NIF-MabCon to the DMM knee. The lack of specific binding in these groups showed that nanosome accumulation is not the result of an inflammatory process. No binding was observed 3 days after sham surgery in mouse that still had inflammation around the suture area. Significant fluorescence is seen only in the surgically destabilized knee of mice that were injected with MabCII nanosomes (Figure 4C). In these mice, surgical destabilization induced the development of arthritis, resulting in the exposure of CII, which allowed the MabCII-targeted nanosomes to access the damaged articular surface. We confirmed after dissection of the knee joint that the fluorescence signal is from the cartilage surface and is neither from the soft tissue nor the blood vessels (the middle panel, tibial cartilage, Figure 4). Safranin-O staining further confirmed these results by showing fissure, clone of cells, and slight loss of proteoglycans in the MabCII nanosomes injected group showing fluorescence signal for the nanosomes.

\section{In vivo binding efficacy of MabCll- targeted nanosomes}

We determined the relationship between articular damage (expressed as an OARSI score) and targeted nanosomes binding (quantified as ROI readings) in order to evaluate the binding efficacy of MabCII nanosomes to the damaged cartilage in vivo (Figures 5 and 6). We observed mice in different stages of surgically induced arthritis in accordance with the OARSI score mentioned in Table 1. As the mean OARSI score increased from 0 to 3 , histological features progressively changed showing loss of proteoglycans, hypocellularity, and surface irregularity. Similarly, IVIS imaging also showed a gradual increase in the fluorescence intensity (radiant efficiency) in ROI as the OARSI score progressed. In the quantification result, the severity of arthritis was increased according to the convention the OARSI score (Figure 6A). Furthermore, we observed increase in the value of ROI, correlated with the increase in OA severity (Figure 6B). ROI can be defined as the region of cartilage damage of the total area of the sample examined. Thus, increase in ROI indicates enhance signal from the MabCII nanosomes, which indicates significant cartilage damage. In this study ROI was able to detect slight damage to the cartilage as was revealed by histology. Therefore, ROI has the potential to be used as a noninvasive standard for quantifying early arthritic change and hence can be used as a helpful tool in determining treatment schedules in patients with early signs of OA.

\section{Tracking of targeted nanosomes in the knee joint}

In vivo live images (by Fluobeam) of fluorescence distribution show that fluorescent-labeled antibodies (MabCII) can be found not only in the synovial cavity but also in whole

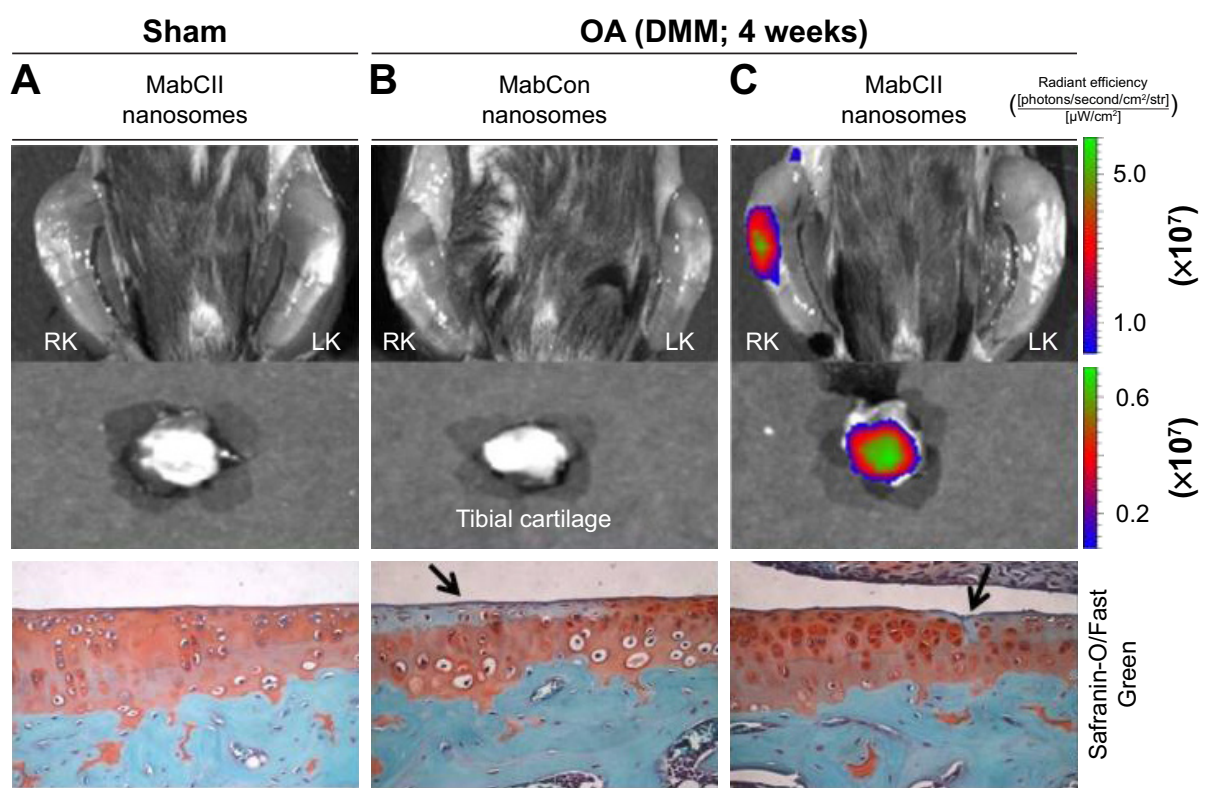

Figure 4 Localization of targeted nanosomes in DMM mice.

Notes: (A) The treatment of targeted nanosome in a mouse that has been subjected to sham surgery of the right knee resulting in no joint damage. (B) and (C) Mice subjected to surgical DMM. The mice in columns $(\mathbf{A})$ and $(\mathbf{C})$ received an injection of MabCll nanosome, and the mouse in column (B) was injected with MabCon nanosome (nontargeted). Significant nanosome binding is only seen in damaged knee of DMM mouse injected with targeted nanosomes (C). Black arrow indicates the damaged cartilage surface as evident by the loss of proteoglycans.

Abbreviations: DMM, destabilization of the medial meniscus; MabClI, monoclonal anti-type II collagen antibodies; MabCon, control antibodies, same subclass mouse lgG; OA, osteoarthritis; RK, right knee joint; LK, left knee joint. 

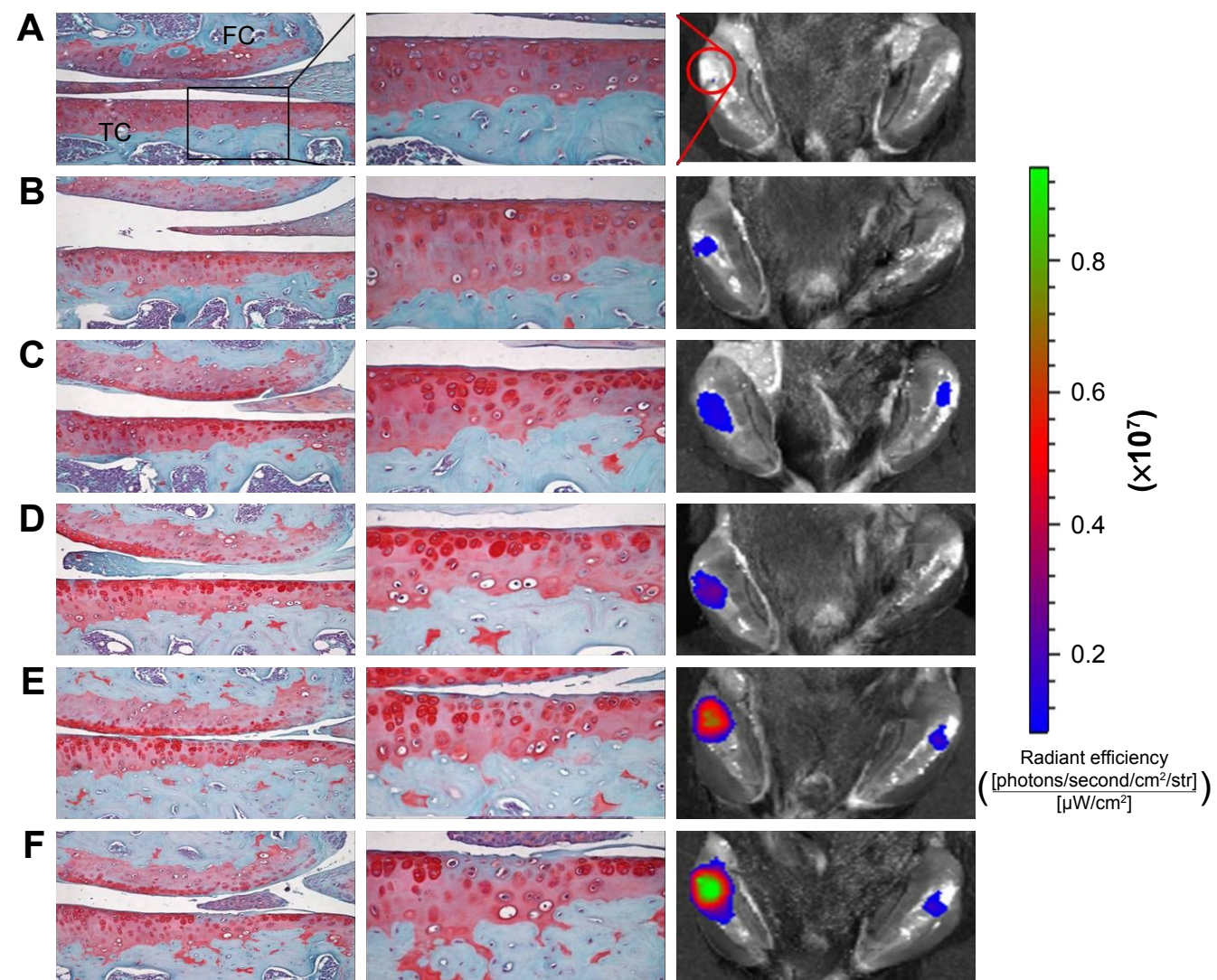

$\mathbf{F}$

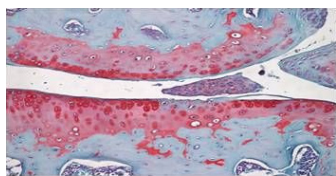

Safranin-O/

Fast Green $(5 x)$

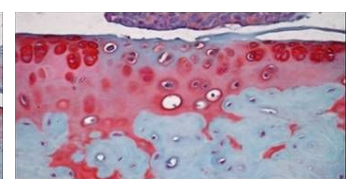

Safranin-O/

Fast Green $(20 x)$

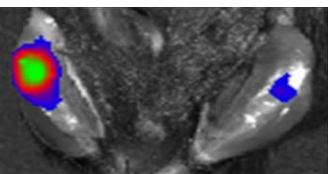

Fluorescence imaging

Figure 5 Histopathology and IVIS scanned images of DMM mouse knee joints.

Notes: (Left) Whole knee joint images, (middle) tibial plateau images, and (right) IVIS imaging of the mouse after injection of targeted nanosomes. Sections of the knee joint were stained with Safranin-O/Fast Green and evaluated histopathologically (left and middle). (A) Sham and (B-F) mice in different stages of development of surgically induced arthritis as described in Table I. Distinct increases in IVIS fluorescence intensity can be observed as the severity of arthritis increased from $\mathbf{A}$ to $\mathbf{F}$. ROI quantification calculated as radiant efficiency.

Abbreviations: DMM, destabilization of the medial meniscus; FC, femoral condyle; ROI, region of interest; TC, tibial condyle.

body images within 3 hours after injection. This imaging was performed only 3 hours after early distribution using skinned mouse samples (Figure 7A). To scan the image after long-term distribution (24 hours) of the nanosomes, we used the IVIS imaging system using normal mouse without being skinned beforehand.

The IVIS imaging shows that, after 24 hours, the fluorescence will no longer be seen in the normal control mouse's
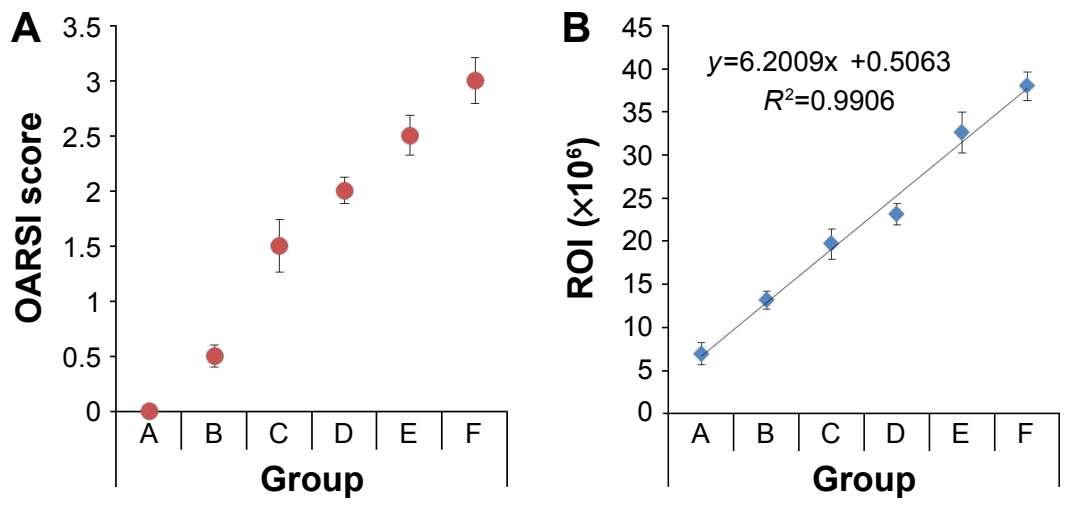

Figure 6 Graphical representation of OARSI score and fluorescent intensity (ROI).

Notes: (A) OARSI score of different groups (A-F) of mice in different stages of arthritis development. (B) Binding of fluorescent nanosomes significantly enhanced with an increase in OARSI score. Group A (sham) and Groups B-F mice were grouped according to the average OARSI score.

Abbreviations: OARSI, Osteoarthritis Research Society International; ROI, region of interest. 


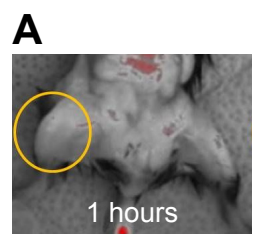

Flurobeam imaging system
B

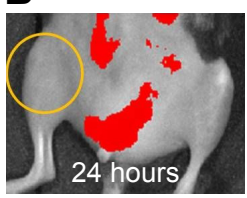

IVIS imaging

system

Figure 7 Tracing of fluorescent targeted-nanosomes in vivo.

Notes: Time course of fluorescence images were shown in normal mouse after systemic injection of fluorescent MabCll nanosomes using near infrared imaging system at I, 2, and 3 hours by Fluobeam (A) and at 24 hours by IVIS scanning (B).

Abbreviation: MabClI, monoclonal anti-type II collagen antibodies.

joint space because the fluorescent antibody did not bind to intact cartilage tissue (Figure 7B).

\section{Discussion}

Proteolysis at the articular surface has been shown to allow access and binding of CII antibodies. ${ }^{4,24,29}$ This observation forms the basis for our antibody-targeted nanosomes binding to the sites of early lesions on the articular cartilage. ${ }^{21}$

Many researchers, up to present day, have preferred ionized labels that bind to the damaged ECM electrostatically and traced using MRI or CT scans. ${ }^{33,34} \mathrm{Hu}$ et al ${ }^{18}$ recently reported using anions, attachable on the cationic GAG, as near-infrared probes. Since CII gets damaged prior to the variation of GAG in the first stage of OA or in parallel with the disappearance of GAG, tracing it will be one of the significant strategies for an early diagnosis. ${ }^{34}$ Although detection of GAG can be used for diagnosis in early OA, the associated method of intra-articular injection is not easy for small animals and it is often difficult to find the damaged site. Furthermore, this technique targets other negatively charged proteins, resulting in nonspecific binding. CII is typically present in the cartilage, and its damage is irreversible, marking the progression of OA..$^{22,24}$ Therefore, the identification of damaged CII could be a potential indicator for the detection and progression of OA. Hence, our strategy is to target the exposed CII. Furthermore, we do believe that the GAG target technique will be a useful validation tool when used in combination with our CII-targeted nanosomes technique, because the increased binding intensity to exposed CII should correlate with a decreased targeting signal from the GAG in progressive early OA. Moreover, GAG targeting is a functional method for the diagnosis of $\mathrm{OA}$, but our CII-targeted nanosome technique is not only diagnostic but may also be advantageous for drug delivery in therapeutic applications.

Our primary goal was to establish an innovative method to identify cartilage damage and to quantitatively measure the progression of OA. We have previously shown that monoclonal antibodies specific to native CII would bind to damaged articular cartilage. ${ }^{21,25,29} \mathrm{We}$ are now proposing to use these antibodies to identify early subclinical lesions and quantify the disease progression in $\mathrm{OA} .{ }^{25}$ We have established the validity of our approach by using a well-characterized animal model of OA, combined with surgical transection for the DMM in mice. ${ }^{26,27}$

It is noteworthy that the experimental mice used in this study do develop arthritis, shown by merely immunization with mouse MabCII without immune or inflammatory complements. ${ }^{29}$ Studies by other workers have suggested that a single antibody injection without adjuvant is not capable of provoking an effective immune response that can result in the development of inflammatory arthritis. ${ }^{35}$

The results of this study have shown that MabCII nanosomes selectively bind to the surface of the eroded articular cartilage, where CII is exposed. ${ }^{21}$ We were able to quantify the degree of damage by using the IVIS imaging technology. ${ }^{36}$ We expected that our technique would identify minimal cartilage damage with great sensitivity and that large lesions would produce amplified signal as a greater quantity of NIF-MabCII would bind to damaged cartilage. Our results showed that the targeted nanosomes were localized to discrete regions of articular cartilage. These regions are marked by OA lesions due to induced DMM. In contrast, NIF-MabCII did not bind to normal articular cartilage (Figure 4). Furthermore, control nanosomes (MabCon or naked) did not bind to either normal or degraded cartilage. We obtained similar results through in vitro testing using cartilage explants damaged by various concentrations of Trypsin-EDTA. The MabCII nanosomes bound specifically to the surface of the damaged cartilage (Figure 3). In addition, we confirmed that MabCII or MabCll-targeted nanosomes encapsulating an NIF dye bound to the damaged cartilage in the DMM mouse model. Notably, quantifications of the degree of cartilage damage observed from fluorescence data matched the stages of OA 
progression in knee joints (Figure 5). After scanning the mice by IVIS, the results were compared with histopathological findings by the OARSI scoring system. The results proved that binding of fluorescent nanosomes increased during early OA development as the OARSI score increased (Figure 6). Fluorescence intensity appeared to be a useful predictor of histopathological damage in mild, early-stage OA (medial cartilage summed OARSI score $<6$ ). Thus, ROI readings had the potential to be used as a noninvasive standard for quantifying early arthritic change. However, long-term studies are required to show whether ROI readings could be used successfully to stage joint damage with higher OARSI scores. These findings may potentially prove helpful to determine treatment schedules in patients with early signs of OA.

The critical advantage of the noninvasive IVIS imaging system using targeted nanosomes is that it does not require that animals be euthanized. This may help in the clinical application of this technique. Though useful in small animal models, it still has a limitation in its application for humans. ${ }^{37}$ This imaging system is a sensitive method of tagging a target of interest; however, detailed anatomical information cannot be obtained. Multifunctional NIF probes, which combine NIF dyes with imaging modalities to provide anatomical information, including MRI and CT, are the next aim of the relatively novel field of CII-targeting NIF imaging technology. ${ }^{38-40}$ Studies comparing MRI with the CII-targeting NIF imaging technique would be beneficial in this regard. While multifunctional NIF probes are still far from being a clinical application, it is anticipated that NIF imaging technology will be an integral part of OA research in the near future.

Furthermore, we can modify the technique to deliver specific therapeutic agents directly to the affected areas of cartilage.

\section{Conclusion}

We established that targeted nanosomes localize to damaged cartilage in vitro. Furthermore, we identified and quantified cartilage damage in vivo for OA induced by DMM in mice using NIF-MabCII and targeted nanosomes. Our technique can detect minimal cartilage damage with a high range of sensitivity. Eventually, MabCII nanosomes with NIF and IVIS imaging could be used as a noninvasive method for diagnosis of the early onset of OA.

\section{Acknowledgments}

This research was supported by a grant from the Korea Health Technology R\&D Project through the Korea Health
Industry Development Institute (KHIDI) and funded by the Ministry of Health \& Welfare, Republic of Korea (grant no HI17C2191). This research also supported by a VA Merit Review award from the Department of Veterans Affairs, an R21 award from NIH (AR060408), and a CTSI award from the UTHSC (KAH). HC also acknowledges the support of Arthritis Foundation Discovery grant and The William and Ella Owens Medical Research Foundation Award.

\section{Author contributions}

All authors contributed toward data analysis, drafting and critically revising the paper and agree to be accountable for all aspects of the work.

\section{Disclosure}

The authors report no conflicts of interest in this work.

\section{References}

1. Woolf AD, Pfleger B. Burden of major musculoskeletal conditions. Bull World Health Organ. 2003;81(9):646-656.

2. Datta P, Zhang Y, Parousis A, et al. High-fat diet-induced acceleration of osteoarthritis is associated with a distinct and sustained plasma metabolite signature. Sci Rep. 2017;7(1):8205.

3. Felson DT, Lawrence RC, Dieppe PA, et al. Osteoarthritis: new insights. Part 1: the disease and its risk factors. Ann Intern Med. 2000;133(8): 635-646.

4. Hollander AP, Heathfield TF, Webber C, et al. Increased damage to type II collagen in osteoarthritic articular cartilage detected by a new immunoassay. J Clin Invest. 1994;93(4):1722.

5. Jasin HE, Taurog JD. Mechanisms of disruption of the articular cartilage surface in inflammation. Neutrophil elastase increases availability of collagen type II epitopes for binding with antibody on the surface of articular cartilage. J Clin Invest. 1991;87(5):1531.

6. Smith GN Jr. The role of collagenolytic matrix metalloproteinases in the loss of articular cartilage in osteoarthritis. Front Biosci. 2006;11: 3081-3095.

7. Chu CR, Williams AA, Coyle CH, Bowers ME. Early diagnosis to enable early treatment of pre-osteoarthritis. Arthritis Res Ther. 2012; 14(3):212.

8. Guermazi A, Niu H, Hayashi D, et al. Prevalence of abnormalities in knees detected by MRI in adults without knee osteoarthritis: population based observational study (Framingham Osteoarthritis Study). BMJ. 2012;345:e5339.

9. Buckwalter JA, Martin JA. Osteoarthritis. Adv Drug Deliv Rev. 2006; 58(2):150-167.

10. Qazi AA, Folkesson J, Pettersen P, Karsdal MA, Christiansen C, Dam EB. Separation of healthy and early osteoarthritis by automatic quantification of cartilage homogeneity. Osteoarthritis Cartilage. 2007; 15(10):1199-1206.

11. Li X, Ma CB, Link TM, et al. In vivo $\mathrm{T} 1 \rho$ and $\mathrm{T} 2$ mapping of articular cartilage in osteoarthritis of the knee using 3T MRI. Osteoarthritis Cartilage. 2007;15:789-797.

12. Burstein D, Gray M. Is MRI fulfilling its promise for molecular imaging of cartilage in arthritis? Osteoarthritis Cartilage. 2006;14: 1087-1090.

13. Glover P, Mansfield P. Limits to magnetic resonance microscopy. Rep Prog Phys. 2002;65:1489.

14. Lamers R, van Nesselrooij J, Kraus V, et al. Identification of an urinary metabolite profile associated with osteoarthritis. Osteoarthritis Cartilage. 2005;13(9):762-768. 
15. Matyas JR, Atley L, Ionescu M, Eyre DR, Poole AR. Analysis of cartilage biomarkers in the early phases of canine experimental osteoarthritis. Arthritis Rheum. 2004;50(2):543-552.

16. Elsaid K, Chichester C. Review: collagen markers in early arthritic diseases. Clin Chim Acta. 2006;365(1-2):68-77.

17. Karsdal MA, Madsen SH, Christiansen C, Henriksen K, Fosang AJ, Sondergaard BC. Cartilage degradation is fully reversible in the presence of aggrecanase but not matrix metalloproteinase activity. Arthritis Res Ther. 2008;10(3):R63.

18. Hu X, Wang Q, Liu Y, et al. Optical imaging of articular cartilage degeneration using near-infrared dipicolylamine probes. Biomaterials. 2014;35(26):7511-7521.

19. Lai W-FT, Chang C-H, Tang Y, Bronson R, Tung C-H. Early diagnosis of osteoarthritis using cathepsin B sensitive near-infrared fluorescent probes. Osteoarthritis Cartilage. 2004;12(3):239-244.

20. Lim N, Meinjohanns E, Meldal M, Bou-Gharios G, Nagase H. In vivo imaging of MMP-13 activity in the murine destabilised medial meniscus surgical model of osteoarthritis. Osteoarthritis Cartilage. 2014; 22:862-868.

21. Cho H, Pinkhassik E, David V, Stuart JM, Hasty KA. Detection of early cartilage damage using targeted nanosomes in a post-traumatic osteoarthritis mouse model. Nanomedicine. 2015;11(4):939-946.

22. Jasin HE, Noyori K, Takagi T, Taurog JD. Characteristics of anti-type II collagen antibody binding to articular cartilage. Arthritis Rheum. 1993; 36(5):651-659.

23. Rousseau J-C, Delmas PD. Biological markers in osteoarthritis. Nat Clin Pract Rheumatol. 2007;3(6):346-356.

24. Noyori K, Koshino T, Takagi T, Okamoto R, Jasin H. Binding characteristics of antitype II collagen antibody to the surface of diseased human cartilage as a probe for tissue damage. J Rheumatol. 1994;21(2): 293-296.

25. Cho H, Bhatti F, Lee $\mathrm{S}$, Brand D, Yi AK, Hasty KA. In vivo dual fluorescence imaging to detect joint destruction. Artif Organs. 2016;40(10): 1009.

26. Glasson S, Blanchet T, Morris E. The surgical destabilization of the medial meniscus (DMM) model of osteoarthritis in the 129/SvEv mouse. Osteoarthritis Cartilage. 2007;15(9):1061-1069.

27. Kim B, Kim D-W, Kim S, et al. Establishment of a reliable and reproducible murine osteoarthritis model. Osteoarthritis Cartilage. 2013; 21(12):2013-2020.

28. Glasson SS, Chambers MG, Van Den Berg WB, Little CB. The OARSI histopathology initiative - recommendations for histological assessments of osteoarthritis in the mouse. Osteoarthritis Cartilage. 2010; 18(suppl 3):S17-S23.
29. Terato K, Hasty KA, Reife RA, Cremer MA, Kang A, Stuart J. Induction of arthritis with monoclonal antibodies to collagen. J Immunol. 1992; 148(7):2103-2108

30. Huwyler J, Wu D, Pardridge WM. Brain drug delivery of small molecules using immunoliposomes. Proc Natl Acad Sci U S A. 1996; 93(24):14164-14169.

31. Rongen HAH, Bult A, van Bennekom WP. Liposomes and immunoassays. J Immunol Methods. 1997;204(2):105-133.

32. Nie Y, Ji L, Ding H, et al. Cholesterol derivatives based charged liposomes for doxorubicin delivery: preparation, in vitro and in vivo characterization. Theranostics. 2012;2(11):1092-1103.

33. Palmer AW, Guldberg RE, Levenston ME. Analysis of cartilage matrix fixed charge density and three-dimensional morphology via contrastenhanced microcomputed tomography. Proc Natl Acad Sci U S A. 2006;103(51):19255-19260.

34. Winalski C, Shortkroff S, Schneider E, Yoshioka H, Mulkern R, Rosen G. Targeted dendrimer-based contrast agents for articular cartilage assessment by MR imaging. Osteoarthritis Cartilage. 2008;16(7): 815-822.

35. Inagawa $\mathrm{K}$, Oohashi $\mathrm{T}$, Nishida $\mathrm{K}$, et al. Optical imaging of mouse articular cartilage using the glycosaminoglycans binding property of fluorescent-labeled octaarginine. Osteoarthritis Cartilage. 2009;17(9): 1209-1218.

36. Holmdahl R, Rubin K, Klareskog L, Larsson E, Wigzell H. Characterization of the antibody response in mice with type II collagen-induced arthritis, using monoclonal anti-type II collagen antibodies. Arthritis Rheum. 1986;29(3):400-410.

37. Leblond F, Davis SC, Valdés PA, Pogue BW. Pre-clinical whole-body fluorescence imaging: Review of instruments, methods and applications. J Photochem Photobiol B. 2010;98(1):77-94.

38. Yuan J, Yi X, Yan F, et al. Near-infrared fluorescence imaging of prostate cancer using heptamethine carbocyanine dyes. Mol Med Rep. 2015;11(2):821-828

39. Qiao X-F, Zhou J-C, Xiao J-W, Wang Y-F, Sun L-D, Yan C-H. Triplefunctional core-shell structured upconversion luminescent nanoparticles covalently grafted with photosensitizer for luminescent, magnetic resonance imaging and photodynamic therapy in vitro. Nanoscale. 2012;4:4611-4623.

40. Kim JS, Kim Y-H, Kim JH, et al. Development and in vivo imaging of a PET/MRI nanoprobe with enhanced NIR fluorescence by dye encapsulation. Nanomedicine. 2012;7(2):219-229.
International Journal of Nanomedicine

\section{Publish your work in this journal}

The International Journal of Nanomedicine is an international, peerreviewed journal focusing on the application of nanotechnology in diagnostics, therapeutics, and drug delivery systems throughout the biomedical field. This journal is indexed on PubMed Central, MedLine, CAS, SciSearch $®$, Current Contents ${ }^{\circledR} /$ Clinical Medicine,

\section{Dovepress}

Journal Citation Reports/Science Edition, EMBase, Scopus and the Elsevier Bibliographic databases. The manuscript management system is completely online and includes a very quick and fair peer-review system, which is all easy to use. Visit http://www.dovepress.com/ testimonials.php to read real quotes from published authors. 